\title{
ON THE NUMBER OF VANISHING CYCLES IN LEFSCHETZ FIBRATIONS
}

\author{
ANDRÁs I. STIPSICZ
}

\begin{abstract}
We prove a lower bound for the number of (nonseparating) vanishing cycles of a genus- $g$ Lefschetz fibration. We also show that a fiber sum $X \#{ }_{f} X$ for a Lefschetz fibration $X \rightarrow S^{2}$ is a minimal symplectic 4-manifold.
\end{abstract}

\section{Statement of results}

Assume that $f: X \rightarrow S^{2}$ is a relatively minimal genus- $g$ Lefschetz fibration with $s$ separating and $n$ nonseparating vanishing cycles. (For definitions of the above notions see Section 2; the reader is also advised to turn to [GS] or [La] for further reference. In our subsequent disscussions we will always assume that $f$ is injective on its critical set.) The fibration is called trivial if $s=n=0$, in this case $X$ is diffeomorphic to $S^{2} \times \Sigma_{g}$ hence it also admits a ruling over the genus- $g$ surface $\Sigma_{g}$. In the following we will prove a bound for $n$ :

Theorem 1.1. If $f: X \rightarrow S^{2}$ is as above and the fibration is nontrivial then

$$
\frac{1}{5}(4 g+2) \leq n
$$

In particular, a genus-g Lefschetz fibration has at least $\frac{1}{5}(4 g+2)$ singular fibers.

\section{Remarks 1.2.}

(a) The above theorem, in particular, shows that $n>0$ - a results earlier found by T.-J. Li [L2] and I. Smith [ABKP]. Since in proving Theorem 1.1 we need $n>0$, in the following we will show an alternative proof for it, cf., Theorem 1.3.

(b) In [C] Cadavid shows genus- $g$ Lefschetz fibrations over $S^{2}$ with $2 g+10$ (and $2 g+4$ in the even $g$ case) singular fibers. In conjunction with Theorem 1.1 this shows that the minimal number $N(g, 0)$ of singular fibers of a genus- $g$ Lefschetz fibration over $S^{2}$ (the surface of genus 0 ) satisfies $\frac{1}{5}(4 g+2) \leq$ $N(g, 0) \leq 2 g+10$. For the determination of $N(g, h)$ (the minimal number of singular fibers in a genus- $g$ Lefschetz fibration over a Riemann surface of genus $h$ ) in case $h>0$ see [KO].

Received February 10, 1999. 
(c) By applying a refined version of Theorem 2.6 (due to Cadavid [C]) we can show that, in fact,

$$
\frac{6}{5}\left(2 g-b_{1}(X)\right)+\frac{4}{5}(g-1) \leq n .
$$

Note that for a genus- $g$ Lefschetz fibration over $S^{2}$ we always have $b_{1} \leq$ $2 g$; once $X \rightarrow S^{2}$ is nontrivial - according to Theorem 1.3 - we have $b_{1}(X)<2 g$.

In the proof of Theorem 1.1 we will use the fact that $n>0$ for a nontrivial Lefschetz fibration; for sake of completeness we give a proof of this statement as well. More precisely, we show the following:

Theorem 1.3. If $X$ is a genus- $g$ Lefschetz fibration with $s$ separating and $n$ nonseparating vanishing cycles, then $s \leq 5 n$. Consequently $n=0$ implies $s=0$, i.e., the triviality of the fibration.

Corollary 1.4. If $X \neq S^{2} \times \Sigma_{g}$ (i.e., the fibration has vanishing cycles) then $n>0$.

The proof of Theorem 1.3 relies on the fact that a relatively minimal Lefschetz fibration over a Riemann surface of positive genus is minimal [S], cf., Theorem 2.7 and Remark 2.8(c). By similar arguments a corresponding statement can be shown for fibrations over $S^{2}$; this statement is interesting in its own right (and leads us to the proof of Theorem 1.1).

Theorem 1.5. If $f: X \rightarrow S^{2}$ is a relatively minimal genus-g Lefschetz fibration, then the fiber sum $X \#_{f} X$ is minimal (as a symplectic 4-manifold).

After giving the basic definitions concerning Lefschetz fibrations, in Section 2 we will give the necessary technical background for the proofs of the above theorems - proofs of some of the theorems applied will be sketched. In Section 3 we give the proofs of the theorems announced above.

\section{Methods and theorems applied in the proofs}

A map $f: X \rightarrow \Sigma$ between the (closed, oriented) 4- and 2-manifolds $X$ and $\Sigma$ is a Lefschetz fibration if $d f$ is onto except at finitely many points $\left\{p_{1}, \ldots, p_{k}\right\}=$ $C$ around which there are orientation preserving charts $U_{i}(i=1, \ldots, k)$ such that $f$ on $U_{i}$ can be modeled by the map $g\left(z_{1}, z_{2}\right)=z_{1}^{2}+z_{2}^{2}$. Any such map admits a perturbation which is still a Lefschetz fibration (in the above sense) with the additional property that $f \mid C$ is injective, i.e., $f\left(p_{i}\right)=f\left(p_{j}\right)$ implies $p_{i}=p_{j}$. (In the following we will always assume that $f$ is injective on the set $C$ of its critical points.) The genus of the Lefschetz fibration $f: X \rightarrow \Sigma$ is by definition the genus of its generic fiber $f^{-1}(t)$ for some $t \in \Sigma-C$. A Lefschetz fibration $f: X \rightarrow \Sigma$ is relatively minimal if there is no fiber $f^{-1}(t)$ containing a sphere of self-intersection -1 . Taking an arc $\gamma_{i}:[0,1] \rightarrow \Sigma$ with $\gamma_{i}(0)=\sigma$ and $\gamma_{i}(1)=f\left(p_{i}\right)$ (with $\sigma \in \Sigma$ fixed) a simple closed curve $v_{i}$ - called a vanishing cycle - in $f^{-1}(\sigma)$ can be identified: This is the curve which gets 
collapsed to a point when travelling from $\sigma$ to $f\left(p_{i}\right)$ along $\gamma_{i}$. The singular fiber $f^{-1}\left(f\left(p_{i}\right)\right)$ is called separating or nonseparating according to the fact whether the corresponding vanishing cycle $v_{i}$ separates (i.e., homologically trivial) or does not separate (i.e., homologically nontrivial) in $f^{-1}(\sigma)$.

\section{Remarks 2.1.}

(a) Note that the vanishing cycle $v_{i}$ itself depends on the chosen path $\gamma_{i}$. It is not very hard to show, however, that its separating property does not depend on this choice.

(b) In fact, it can be shown that a singular fiber $f^{-1}\left(f\left(p_{i}\right)\right)$ is the immersed image of a (not necessarily connected) Riemann surface $S$ with a single (transverse) double point and $f^{-1}\left(f\left(p_{i}\right)\right)$ is nonseparating iff $S$ is connected. (If $f^{-1}\left(f\left(p_{i}\right)\right)$ corresponds to a separating vanishing cycle, then $S$ has two components and the image of each component in $X$ has selfintersection -1.)

(c) Relative minimality means that although $X$ might contain an embedded -1-sphere, by blowing it down we ruin the fibration given on $X$. Not relatively minimal Lefschetz fibrations can be blown down while preserving the fiber structure. (Another way to detect relative minimality is that in such a fibration there is no homotopically trivial vanishing cycle.) A symplectic 4-manifold $X$ is called minimal if it does not contain any embedded -1-sphere.

(d) By weakening the definition of Lefschetz fibration - by dropping the requirement that the charts $U_{i}$ should be orientation preserving — we get a related notion, called achiral Lefschetz fibration. Such (more general) fibrations, however, do not necessarily admit symplectic structures, hence most of the arguments presented in this paper do not apply for achiral Lefschetz fibrations.

Before giving the proofs of the theorems announced in Section 1, we quickly review the necessary background. We begin with the description of the connection between Lefschetz fibrations and symplectic structures on 4-manifolds.

Theorem 2.2. (Donaldson, [D] and Gompf, [GS]) If $(X, \omega)$ is a symplectic 4-manifold, then there exists a positive integer $n$ such that the $n$-fold blow-up $X \# n \overline{\mathbf{C P}^{2}}$ admits a Lefschetz fibration $X \# n \overline{\mathbf{C P}^{2}} \rightarrow S^{2}$. Conversely, a genus- $g$ Lefschetz fibration $f: X \rightarrow \Sigma$ with fiber-genus $g \geq 2$ over a Riemann surface $\Sigma$ admits a symplectic structure.

Consequently, in studying Lefschetz fibrations we can always assume that the 4-manifold under consideration is symplectic, hence the powerful methods of symplectic topology apply. At the same time, information about the topological behaviour of Lefschetz fibrations might lead us to a better understanding of the topology of symplectic 4-manifolds. (We will always assume that the genus of the fiber in the fibrations considered is $\geq 2$, i.e., Theorem 2.2 applies and equips the total space with a symplectic structure. The $g \leq 1$ case is fairly well-understood, cf. [GS], for example.) 
In studying the topology of symplectic 4-manifolds, theorems of Taubes turn out to be extremely powerful. In the following we will only outline the few special cases of his theory relating almost-complex geometric properties of a symplectic 4-manifold to its differential topological invariants.

Theorem 2.3. (Taubes, [T2]) If $(X, \omega)$ is a symplectic 4-manifold with $b_{2}^{+}(X)>1$ and $\Sigma \subset X$ is an embedded sphere with $[\Sigma]^{2}=-1$, then for a generic almost-complex structure $J$ the homology class $[\Sigma]$ can be represented by a J-holomorphic sphere $C$.

Theorem 2.4. (Taubes, [T2] and Liu, [Liu]) If $(X, \omega)$ is a minimal symplectic 4-manifold then $c_{1}^{2}(X, \omega) \geq 0$ holds unless $X$ is a ruled surface, i.e., diffeomorphic to an $S^{2}$-bundle over a Riemann surface $\Sigma$. (Note that in this latter case $b_{2}^{+}(X)=1$.)

Theorem 2.5. (Li, [L1]) If $X$ is the blow-up of a minimal symplectic 4-manifold which is not rational or ruled, and $e_{i}(i=1, \ldots, n)$ are the homology classes of the exceptional spheres of the blow-ups, then these $e_{i}$ 's are the only homology classes which can be represented by smoothly embedded spheres of square -1 . Consequently, $\Sigma_{1}, \Sigma_{2}$ symplectic -1 -spheres in $X$ cannot satisfy $\left[\Sigma_{1}\right] \cdot\left[\Sigma_{2}\right]>0$ once $b_{2}^{+}(X)>1$.

Theorem 2.6. (Ozbagci, [Ob]) If $X \rightarrow S^{2}$ is a Lefschetz fibration with s separating and $n$ nonseparating vanishing cycles, then for the signature of $X$ we have $\sigma(X) \leq n-s$. Moreover, if $n>0$, then $\sigma(X) \leq n-s-2$.

Theorem 2.7. ([S]) If $X \rightarrow \Sigma$ is a relatively minimal Lefschetz fibration over the Riemann surface $\Sigma$ with positive genus, then $X$ is a minimal symplectic 4manifold. Since for such a fibration we have $b_{2}^{+}(X)>1$, Theorem 2.4 implies that $c_{1}^{2}(X) \geq 0$.

In the following remark we briefly indicate the proofs of the above theorems.

\section{Remarks 2.8.}

(a) The proofs of Theorems 2.3 and 2.4 follow from Taubes' argument showing that if the Seiberg-Witten invariant $S W_{X}\left(c_{1}(X, \omega)+2 K\right)$ of the symplectic 4-manifold $X$ with $b_{2}^{+}(X)>1$ is nonzero, then (for a generic almostcomplex structure) $P D(K) \in H_{2}(X ; \mathbf{Z})$ can be represented by a pseudoholomorphic curve. By the blow-up formula it already proves Theorem 2.3; since (according to [T1]) $S W_{X}\left(-c_{1}(X, \omega)\right)= \pm 1, c_{1}^{2}(X, \omega) \geq 0$ follows once $(X, \omega)$ is minimal, i.e., the pseudo-holomorphic representative of $-c_{1}(X, \omega)$ has no sphere component. The characterization of minimal symplectic manifolds with $c_{1}^{2}(X)<0$ (due to Liu [Liu]) involves a detailed analysis of the change of $S W_{X}$ under certain perturbations together with symplectic geometric results of McDuff.

(b) It can be shown [Ob] that every vanishing cycle (corresponding to a 2handle in an appropriate handle decomposition of $X$ ) contributes 0 or \pm 1 to $\sigma(X)$. By showing that a separating vanishing cycle necessary contributes 
-1 , Ozbagci concluded $\sigma(X) \leq n-s$. Noting that the first nonseparating vanishing cycle contributes 0 (since it necessarily kills the corresponding element in the first chain group of $X$ ), and realizing that by Poincaré duality the corresponding element in the third chain group has to be killed by a 2 -handle as well, we arrive to $\sigma(X) \leq n-s-2$ once $n>0$. Since the dimension of the subspace of $H_{1}(F ; \mathbf{R})$ spanned by the vanishing cycles is $2 g-b_{1}(X)$ (here $F$ is the generic fiber of the fibration), the same argument yields $\sigma(X) \leq n-s-2\left(2 g-b_{1}(X)\right)$, cf., [C].

(c) In proving Theorem 2.7, for nonminimal $X$ (according to Theorem 2.3) the -1 -sphere can be represented by a pseudo-holomorphic sphere $C$. Restricting the projection $f: X \rightarrow \Sigma$ to that sphere we get a holomorphic map $f: C \rightarrow \Sigma$, which obviously has degree 0, i.e., $C$ is in the fiber. By assuming that $f$ is injective on its critical set (which can be arranged by a slight perturbation of the fibration), we know that a sphere contained by a fiber has square -1 . Consequently if $X$ is nonminimal, then the fibration is not relatively minimal; for details see $[\mathrm{S}]$.

\section{The proofs}

Proof of Theorem 1.3. Suppose that $X \rightarrow S^{2}$ is a relatively minimal genus- $g$ Lefschetz fibration with $s$ separating and $n$ nonseparating vanishing cycles. For the Euler characteristic $\chi(X)$ we have $\chi(X)=4-4 g+n+s$; by definition $c_{1}^{2}(X)=3 \sigma(X)+2 \chi(X)=3 \sigma(X)+2(4-4 g+n+s)$. Applying Theorem 2.6 we get that

$$
c_{1}^{2}(X) \leq 3(n-s)+2(4-4 g+n+s)=5 n-s+8-8 g .
$$

Take the fiber sum $Y=X \#_{f} \Sigma_{g} \times T^{2}$ of $X$ with the trivial genus- $g$ Lefschetz fibration over the torus $T^{2}$. The resulting symplectic 4-manifold $Y$ obviously fibers over $T^{2}$, and since $X$ is relatively minimal, by Theorem $2.7 Y$ is a minimal symplectic 4-manifold with $b_{2}^{+}(Y)>1$. Consequently $c_{1}^{2}(Y)$ (which is equal to $\left.c_{1}^{2}(X)+8 g-8\right)$ is nonnegative by Theorem 2.4, implying that

$$
8-8 g \leq c_{1}^{2}(X) \text {. }
$$

The two inequalities above imply $0 \leq 5 n-s$ which proves Theorem 1.3.

Before proceeding further, we prove a helpful topological lemma regarding $b_{2}^{+}$ of the fiber sum $X \#_{f} X$.

Lemma 3.1. For the fiber sum $X \#_{f} X$ we have $b_{2}^{+}\left(X \#_{f} X\right)=2 b_{2}^{+}(X)-1+$ $\left(2 g-b_{1}(X)\right)$. In particular, if the fibration $X \rightarrow S^{2}$ admits singular fibers then $b_{2}^{+}\left(X \#_{f} X\right) \geq 2$.

Proof. Since the vanishing cycles of $X \rightarrow S^{2}$ and of $X \#{ }_{f} X \rightarrow S^{2}$ are the same (for $X \#_{f} X$ we just repeat the same set of cycles again), we obviously get that $b_{1}(X)=b_{1}\left(X \#_{f} X\right)$. The facts $\sigma\left(X \#_{f} X\right)=2 \sigma(X)$ and $\chi\left(X \#_{f} X\right)=$ 
$2 \chi(X)-2(2-2 g)$ together with the definitions $\sigma(X)=b_{2}^{+}(X)-b_{2}^{-}(X)$ and $\chi(X)=2-2 b_{1}(X)+b_{2}^{+}(X)+b_{2}^{-}(X)$ imply the result.

By Theorem 1.3, if $X \rightarrow S^{2}$ is nontrivial, then it admits nonseparating vanishing cycles, i.e., for those fibrations $b_{1}(X)<2 g$. (The first 2-handle attached along a nonseparating vanishing cycle kills the corresponding first homology element.) Since $b_{2}^{+}(X) \geq 1$ by the existence of a symplectic structure on $X$, the second assertion of the lemma easily follows.

Now we are in the position to give the proof of Theorem 1.1. It relies on Theorem 1.5, the proof of which will be given later.

Proof of Theorem 1.1. Assuming Theorem 1.5, roughly the same idea which proved Theorem 1.3 above, provides the proof of Theorem 1.1 once we show

Lemma 3.2. Suppose that $X \rightarrow S^{2}$ is a relatively minimal genus-g Lefschetz fibration. If $c_{1}^{2}(X)<4-4 g$ then $c_{1}^{2}(X)=8-8 g$ and $X=S^{2} \times \Sigma_{g}$.

Proof. Assume that $c_{1}^{2}(X)<4-4 g$ and suppose that $X$ is not the trivial fibration; hence by Theorem 1.3 we have that $n>0$. Consider the fiber sum $X \#_{f} X$. By Lemma 3.1 (and since $X \rightarrow S^{2}$ is nontrivial) we have $b_{2}^{+}\left(X \#_{f} X\right) \geq$ 2 ; on the other hand $c_{1}^{2}(X)<4-4 g$ shows that $c_{1}^{2}\left(X \#_{f} X\right)=2 c_{1}^{2}(X)+8 g-$ $8<0$. Since Theorem 1.5 shows that $X \#_{f} X$ is minimal, we found a minimal sympletcic manifold with $b_{2}^{+}(X)>1$ and $c_{1}^{2}(X)<0$. This, however, contradicts Theorem 2.4, showing that if $c_{1}^{2}(X)<4-4 g$, then $X$ is the trivial genus- $g$ fibration and so $c_{1}^{2}(X)=8-8 g$.

Now the proof of Theorem 1.1 is easy: Since by assumption $X \neq S^{2} \times \Sigma_{g}$, according to Lemma 3.2 above we have $c_{1}^{2}(X) \geq 4-4 g$. By Theorem 1.3 we also know that $n>0$, so the relevant result of Theorem 2.6 tells us that $\sigma(X) \leq n-s-2$. The argument given for the proof of Theorem 1.3 now modifies to the inequality

$$
4-4 g \leq 5 n-s-6+8-8 g,
$$

implying $s+4 g+2 \leq 5 n$. Since $s \geq 0$, this latter inequality obviously yields the result.

\section{Remarks 3.3.}

(a) Lemma 3.2 is sharp in the sense that there are genus- $g$ Lefschetz fibrations $X_{g} \rightarrow S^{2}$ with $c_{1}^{2}\left(X_{g}\right)=4-4 g$ : Take the double branched cover of $S^{2} \times S^{2}$ (presented as the trivial $S^{2}$-fibration over $S^{2}$ ) branched along two fibers and $2 g+2$ sections. The resolution of the manifold given this way will provide an example of a relatively minimal genus- $g$ Lefschetz fibration with $c_{1}^{2}=4-4 g$ [GS]. Note that all these examples are blow-ups of the complex projective plane; in fact, $X_{g} \approx \mathbf{C P}^{2} \#(4 g+5) \overline{\mathbf{C P}^{2}}$. (According to a recent result of Li [L3], if $X$ admits a genus- $g$ Lefschetz fibration and it is not the blow-up of a ruled surface (for example, if $b_{2}^{+}(X)>1$ ), then in fact $c_{1}^{2}(X) \geq 2-2 g$.) 
(b) It is expected that in a generic Lefschetz fibration there are no separating vanishing cycles; hence we cannot expect to improve our bounds by finding lower bounds for $s$.

(c) By invoking $\sigma(X) \leq n-s-2\left(2 g-b_{1}(X)\right)$ (cf., Remark 2.8(b)), the same argument as above yields $\frac{6}{5}\left(2 g-b_{1}(X)\right)+\frac{4}{5}(g-1) \leq n$, cf. Remark 1.2(c).

Finally we turn to the proof of Theorem 1.5. In the proof we will appeal to Theorem 2.5 of $\mathrm{Li}$; an alternative way to conclude the proof is outlined in Remark 3.4.

Proof of Theorem 1.5. Equip $X$ with an almost-complex structure for which the fibers of $X \rightarrow S^{2}$ are pseudo-holomorphic. The fiber sum $X \#_{f} X$ can be given as the double branched cover of $X$ along a pair of generic fibers [GS] — in this way $X \#_{f} X$ comes with an almost-complex structure $J$ (inherited from $X$ ) and a pseudo-holomorphic involution $\tau: X \#_{f} X \rightarrow X \#_{f} X$. For this involution $\left(X \#_{f} X\right) / \tau=X$ and the fixed point set $\operatorname{Fix}(\tau)$ can be identified with a pair of regular fibers (the inverse image of the branch locus of the branched cover $\left.X \#_{f} X \rightarrow X\right)$.

Now suppose that $X \#_{f} X$ is nonminimal, i.e., it contains an embedded sphere $\Sigma$ of self-intersection -1 . Represent $[\Sigma]$ with a $J$-holomorphic curve $C$ and consider $\tau(C)$. Since $\tau$ is $J$-holomorphic, $\tau(C)$ is a $J$-holomorphic curve.

If $\tau(C)=C$ then $C$ lifts from a curve in $X$. All such lifts have even squares unless the curve is contained in the branch locus. In our case, however, $[C]^{2}=-1$ and the branch locus (a pair of regular fibers) contains no closed curve of nonzero self-intersection; hence we conclude that $\tau(C) \neq C$. By $[\mathrm{M}]$ we know that in case $\tau(C) \neq C$, every intersection of the two curves is positive - in particular, $[\tau(C)] \cdot[C] \geq 0$. Since $C \cap \operatorname{Fix}(\tau) \subset C \cap \tau(C)$, the equation $[\tau(C)] \cdot[C]=0$ implies that $C$ is disjoint from the generic fiber (which is part of $\operatorname{Fix}(\tau)$ ). In this case, by restricting the projection map $f: X \#_{f} X \rightarrow S^{2}$ to the sphere component $C_{1}$ of $C$, we get a holomorphic map $f \mid C_{1}: C_{1} \rightarrow S^{2}$ of degree 0 . Consequently $C_{1}$ is in a fiber of $X \#_{f} X$; now standard argument gives a contradiction with the relative minimality of $X \rightarrow S^{2}$, cf. [S] and Remark 2.8(c). Finally, if $C \cap \tau(C) \neq \emptyset$ (that is, $[\tau(C)] \cdot[C]>0$ ), we found two symplectic -1 -spheres $\Sigma$ and $\tau(\Sigma)$ in $X \#_{f} X$ (with $b_{2}^{+}\left(X \#_{f} X\right)>1$ by Lemma 3.1) intersecting each other positively, which contradicts Theorem 2.5. According to the above contradictions we showed that $X \#_{f} X$ cannot contain any embedded -1 -sphere, hence the proof of Theorem 1.5 is complete.

Remark 3.4. For the sake of completeness we sketch an argument showing that in a symplectic 4-manifold $X$ with $b_{2}^{+}(X)>1$ symplectic spheres of square -1 cannot intersect each other positively. Assume, in the contrary, that spheres $\Sigma_{1}, \Sigma_{2} \subset X$ satisfy $\left[\Sigma_{1}\right]^{2}=\left[\Sigma_{2}\right]^{2}=-1$ and $\left[\Sigma_{1}\right] \cdot\left[\Sigma_{2}\right]=k>0$ (with orientations compatible with the symplectic structures). For a generic almost-complex structure $J$ represent $\left[\Sigma_{1}\right],\left[\Sigma_{2}\right]$ by $J$-holomorphic spheres intersecting transversally in $k$ points. Blowing $\Sigma_{1}$ down, $\Sigma_{2}$ defines an immersion $\varphi$ of $S^{2}$ into the blown 
down manifold $Y$ with an ordinary $k$-tuple point. Since by blowing $\varphi\left(S^{2}\right)$ back again we get $\Sigma_{2}$, we have $\left[\varphi\left(S^{2}\right)\right]-k\left[\Sigma_{1}\right]=\left[\Sigma_{2}\right]$. By perturbing $\varphi: S^{2} \rightarrow Y$ slightly we get an immersion $\tilde{\varphi}: S^{2} \rightarrow Y$ with $\frac{k(k-1)}{2}$ positive double points and $\left[\tilde{\varphi}\left(S^{2}\right)\right]^{2}=\left[\varphi\left(S^{2}\right)\right]^{2}=k^{2}-1$. Since $b_{2}^{+}(Y)=b_{2}^{+}(X)>1$, this configuration contradicts the adjunction formula of Fintushel and Stern [FS] found for immersed spheres in symplectic 4-manifolds with $b_{2}^{+}>1$. Note that the above argument also shows that a symplectic 4 -manifold with $b_{2}^{+}>1$ admits a unique minimal model. The result of Li quoted above, in fact, shows that the minimal model is always unique unless the symplectic 4-manifold is the blow-up of a rational or ruled surface, cf. [L1].

\section{Acknowledgement}

The author would like to thank Ludmil Katzarkov, Burak Ozbagci and Ron Stern for helpful conversations.

\section{References}

[ABKP] J. Amorós, F. Bogomolov, L. Katzarkov and T. Pantev, Symplectic Lefschetz fibrations with arbitrary fundamental groups, preprint.

[C] C. Cadavid, Ph.D. dissertation, UT Austin, 1998.

[D] S. Donaldson, Lefschetz fibrations in symplectic geometry, Proceedings of the International Congress of Mathematicians, Vol. II (Berlin, 1998), Doc. Math. (1998), Extra Vol. II, 309-314.

[FS] R. Fintushel and R. Stern, Immersed spheres in 4-manifolds and the immersed Thomconjecture, Turkish J. Math. 19 (1995), 145-157.

[GS] R. Gompf and A. Stipsicz, 4-manifolds and Kirby calculus, book to appear.

[KO] M. Korkmaz and B. Ozbagci, Minimal number of singular fibers in a Lefschetz fibration, preprint.

[La] K. Lamotke, The topology of complex projective varieties after S. Lefschetz, Topology 20 (1981), 15-51.

[L1] T.-J. Li, Smoothly embedded spheres in symplectic 4-manifolds, Proc. Amer. Math. Soc. 127 (1999), 609-613.

[L2] L Lefschetz fibrations and symplectic 4-manifolds, preprint.

[L3] private communication.

[Liu] A. Liu, Some new applications of the general wall crossing formula, Math. Res. Lett. 3 (1996), 569-585.

[M] D. McDuff, The local behaviour of holomorphic curves in almost-complex 4manifolds, J. Diff. Geom. 34 (1991), 311-358.

[Ob] B. Ozbagci, Signatures of Lefschetz fibrations, preprint.

[S] A. Stipsicz, Chern numbers of certain Lefschetz fibrations, Proc. Amer. Math. Soc., to appear.

[T1] C. Taubes, The Seiberg-Witten invariants and symplectic forms, Math. Res. Lett. 1 (1994), 809-822.

[T2] $\quad S W \Longrightarrow G r$ : From the Seiberg-Witten equations to pseudo-holomorphic curves, J. Amer. Math. Soc. 9 (1996), 845-918.

Department of Analysis, Elte TTK, 1088. Múzeum krt. 6-8., Budapest, Hungary and Department of Mathematics, University of California, Irvine, 92697

E-mail address: stipsicz@cs.elte.hu and astipsic@math.uci.edu 